\title{
X-type Soliton, Resonance Y-type Soliton and Hybrid Solutions of a (2+1)-dimensional Hirota-Satsuma-Ito System for the Shallow Water Waves
}

Yuan Shen ( $\nabla$ yuanshen@bupt.edu.cn )

Beijing University of Posts and Telecommunications https://orcid.org/0000-0002-8858-5140

Bo Tian

Beijing University of Posts and Telecommunications

Tian-Yu Zhou

Beijing University of Posts and Telecommunications

Xiao-Tian Gao

Beijing University of Posts and Telecommunications

\section{Research Article}

Keywords: Shallow water waves, (2+1)-dimensional Hirota-Satsuma-lto system, Sym- bolic computation, Hirota method, X-type soliton solutions, Resonance Y-type soliton solutions, Hybrid solutions

Posted Date: July 30th, 2021

DOl: https://doi.org/10.21203/rs.3.rs-751414/v1

License: (9) This work is licensed under a Creative Commons Attribution 4.0 International License. Read Full License 


\title{
X-type soliton, resonance Y-type soliton and hybrid solutions of a $(2+1)$-dimensional Hirota-Satsuma-Ito system for the shallow water waves
}

\author{
Yuan Shen, Bo Tian, Tian-Yu Zhou, Xiao-Tian Gao
}

State Key Laboratory of Information Photonics and

Optical Communications, and School of Science, Beijing University of Posts

and Telecommunications, Beijing 1008\%6, China

\begin{abstract}
Water waves are observed in the rivers, lakes, oceans, etc. Under investigation in this paper is a $(2+1)$-dimensional Hirota-Satsuma-Ito system arising in the shallow water waves. Via the Hirota method and symbolic computation, we derive some Xtype and resonance Y-type soliton solutions. We also work out some hybrid solutions consisting of the resonance Y-type solitons, solitons, breathers and lumps. Graphics we present reveal that the hybrid solutions consisting of the resonance Y-type solitons and solitons/breathers/lumps describe the interactions between the resonance Y-type solitons and solitons/breathers/lumps, respectively. The obtained results rely on the water-wave coefficient in that system.
\end{abstract}

Keywords: Shallow water waves; $(2+1)$-dimensional Hirota-Satsuma-Ito system; Symbolic computation; Hirota method; X-type soliton solutions; Resonance Y-type soliton solutions; Hybrid solutions

*Corresponding author, with e-mail address as yuanshen@bupt.edu.cn 


\section{Introduction}

Waves, one of the most common natural phenomena, have attracted people's attention [1-6]. It has been known that mechanical waves consist of the water waves, seismic waves, sound waves in air, electromagnetic waves, quantum waves, etc [7-12]. Among them, the subject of water waves has been the most fascinating and varied of all areas in the study of wave motions [13]. The core of fluid dynamics in general, especially ocean dynamics, has been the study of the water waves and their various ramifications [13]. It has been a fact that most of the fundamental ideas and results for nonlinear dispersive waves and solitons originated in the investigation of the water waves [14, 15].

Nonlinear evolution equations (NLEEs) have been proposed to model certain nonlinear phenomena in nonlinear optics, fluid mechanics, plasma physics, etc [16-22]. As some NLEEs, shallow-water equations have been used to depict the horizontal structure of an atmosphere and the evolution of an incompressible fluid [23]. There have been some methods to solve the NLEEs, including the Lie symmetry approach, Bäcklund transformation, Darboux transformation, Hirota method, and so on [24-30].

Solitons, one type of the nonlinear waves, have been found in optics, fluid mechanics, plasma physics and so on [31-33]. Soliton resonance phenomenon has been actively studied, and initially defined as "at the critical angle of intersection, two obliquely directed solitons interact strongly to make a branch soliton from a point at which the wave fronts of the two solitons meet together" [34]. For certain NLEEs, it has been believed that the resonances of solitons might give rise to various types of new excitations such as the breathers, lumps, web solitons, solitonic fissions and fusions, rogue and rational-exponential waves [35].

The Hirota-Satsuma shallow water wave system [36, 37],

$$
\left\{\begin{array}{l}
\varphi_{t}=\varphi_{x x t}+3 \varphi \varphi_{t}-3 \varphi_{x} \psi_{t}-\varphi_{x} \\
\psi_{x}=-\varphi
\end{array}\right.
$$

has been introduced to describe the unidirectional shallow water waves, where $\varphi(x, t)$ and $\psi(x, t)$ are the functions of the variables $x$ and $t$, while the subscripts denote the partial derivatives. For the shallow water waves, Refs. [37-44] have considered the following generalization of Eqn. (1) called the Hirota-Satsuma-Ito (HSI) system:

$$
\left\{\begin{array}{l}
v_{t}+u_{x x t}+3(u w)_{x}+\alpha u_{x}=0 \\
u_{y}=v_{x} \\
u_{t}=w_{x}
\end{array}\right.
$$

with $u(x, y, t), v(x, y, t)$ and $w(x, y, t)$ being the functions of the scaled spatial coordinates $x$, $y$ and temporal coordinate $t, \alpha$ being a real non-zero constant, $u(x, y, t)$ meaning the physical field, while $v(x, y, t)$ and $w(x, y, t)$ representing the potentials of physical field derivatives. 
Via the dependent variable transformations as follows:

$$
u=2(\ln f)_{x x}, \quad v=2(\ln f)_{x y}, \quad w=2(\ln f)_{x t},
$$

Ref. [43] has derived a bilinear form for System (2) as

$$
\left(D_{x}^{3} D_{t}+D_{y} D_{t}+\alpha D_{x}^{2}\right) f \cdot f=0
$$

where $f$ is a real differentiable function of $x, y$ and $t$, while $D_{x}, D_{y}$ and $D_{t}$ are the bilinear operators defined by [36]

$$
\begin{aligned}
& D_{x}^{a_{1}} D_{y}^{a_{2}} D_{t}^{a_{3}}(G \cdot H)= \\
& \left.\quad\left(\frac{\partial}{\partial x}-\frac{\partial}{\partial x^{\prime}}\right)^{a_{1}}\left(\frac{\partial}{\partial y}-\frac{\partial}{\partial y^{\prime}}\right)^{a_{2}}\left(\frac{\partial}{\partial t}-\frac{\partial}{\partial t^{\prime}}\right)^{a_{3}} G(x, y, t) H\left(x^{\prime}, y^{\prime}, t^{\prime}\right)\right|_{x^{\prime}=x, y^{\prime}=y, t^{\prime}=t},
\end{aligned}
$$

with $G(x, y, t)$ being a differentiable function with respect to $x, y$ and $t, H\left(x^{\prime}, y^{\prime}, t^{\prime}\right)$ being a differentiable function of the independent variables $x^{\prime}, y^{\prime}$ and $t^{\prime}$, while $a_{1}, a_{2}$ and $a_{3}$ being the non-negative integers. $N$-soliton solutions for System (2) have been presented as [38]

$$
\begin{aligned}
& u=2(\ln f)_{x x}, \\
& v=2(\ln f)_{x y}, \\
& w=2(\ln f)_{x t}, \\
& f=1+\sum_{\imath=1}^{N} e^{\eta_{\imath}}+\sum_{\imath<\jmath}^{N} A_{\imath \jmath} e^{\eta_{\imath}+\eta_{\jmath}}+\sum_{\imath<\jmath<\ell}^{N} A_{\imath \jmath} A_{\imath \ell} A_{\jmath \ell} e^{\eta_{\imath}+\eta_{\jmath}+\eta_{\ell}}+\cdots+\left(\prod_{\imath<\jmath}^{N} A_{\imath \jmath}\right) e^{\sum_{\imath}^{N} \eta_{\imath},}
\end{aligned}
$$

where

$$
\begin{aligned}
& \eta_{\imath}=k_{\imath} x+p_{\imath} y+\omega_{\imath} t+\zeta_{\imath}, \quad \omega_{\imath}=-\frac{\alpha k_{\imath}^{2}}{k_{\imath}^{3}+p_{\imath}}, \\
& A_{\imath \jmath}=-\frac{\left(k_{\imath}-k_{\jmath}\right)^{3}\left(\omega_{\imath}-\omega_{\jmath}\right)+\alpha\left(k_{\imath}-k_{\jmath}\right)^{2}+\left(p_{\imath}-p_{\jmath}\right)\left(\omega_{\imath}-\omega_{\jmath}\right)}{\left(k_{\imath}+k_{\jmath}\right)^{3}\left(\omega_{\imath}+\omega_{\jmath}\right)+\alpha\left(k_{\imath}+k_{\jmath}\right)^{2}+\left(p_{\imath}+p_{\jmath}\right)\left(\omega_{\imath}+\omega_{\jmath}\right)},
\end{aligned}
$$

with $N$ being a positive integer, $\imath, \jmath, \ell=1,2, \cdots, N, k_{\imath}$ 's, $p_{\imath}$ 's and $\zeta_{\imath}$ 's being the complex constants, $\sum_{\imath<\jmath}^{N}$ implying the summation over all possible combinations of $N$ elements under the condition $\imath<\jmath$, and $\prod_{\imath<\jmath}^{N}$ meaning the product over all possible combinations of $N$ elements under the condition $\imath<\jmath$. There have been some works closely related to System (2): complexiton solutions [37], localized wave interaction solutions [38], rational localized waves and their Absorb-Emit interactions [39], multi-wave, breather wave and hybrid solutions [40], resonant multi-soliton solutions [41], higher-order breathers, lumps and semi-rational solutions [42], lump and lump-soliton solutions [43], Alice-Bob HSI system and its Bäcklund transformation, bilinear form, lump and breather solutions [44].

However, to our knowledge, X-type soliton solutions, resonance Y-type soliton solutions different from those in Ref. [41], and hybrid solutions composed of the resonance Y-type 
solitons and solitons/breathers/lumps for System (2) have not been reported. In Sec. 2, two X-type soliton solutions for System (2) will be obtained based on the Hirota method and symbolic computation. In Sec. 3, resonance Y-type soliton solutions which differ from those in Ref. [41] will be worked out through the Hirota method and symbolic computation. In Sec. 4, hybrid solutions composed of the 2-resonance solitons and two solitons/the first-order breathers/the first-order lumps for System (2) will be presented via the Hirota method and symbolic computation. Section 5 will be our conclusions.

\section{X-type soliton solutions for System (2)}

To search for some X-type soliton solutions for System (2), we consider the two-soliton solutions for System (2) obtained from $N=2$ in $N$-Soliton Solutions (5), i.e.,

$$
\begin{aligned}
& u=2(\ln f)_{x x}, \\
& v=2(\ln f)_{x y}, \\
& w=2(\ln f)_{x t}, \\
& f=1+e^{\eta_{1}}+e^{\eta_{2}}+A_{12} e^{\eta_{1}+\eta_{2}},
\end{aligned}
$$

where

$$
\begin{aligned}
& \eta_{i}=k_{i} x+p_{\imath} y+\omega_{i} t+\zeta_{i}, \quad \omega_{i}=-\frac{\alpha k_{i}^{2}}{k_{i}^{3}+p_{i}}, \\
& A_{12}=-\frac{\left(k_{1}-k_{2}\right)^{3}\left(\omega_{1}-\omega_{2}\right)+\alpha\left(k_{1}-k_{2}\right)^{2}+\left(p_{1}-p_{2}\right)\left(\omega_{1}-\omega_{2}\right)}{\left(k_{1}+k_{2}\right)^{3}\left(\omega_{1}+\omega_{2}\right)+\alpha\left(k_{1}+k_{2}\right)^{2}+\left(p_{1}+p_{2}\right)\left(\omega_{1}+\omega_{2}\right)},
\end{aligned}
$$

while $k_{i}$ 's, $p_{i}$ 's and $\zeta_{i}$ 's $(i=1,2)$ are the real constants. Setting certain conditions on $A_{12}$ in Two-Soliton Solutions (6), we are able to derive two X-type soliton solutions for System (2) as follows:

\section{Type 1.}

$$
\begin{aligned}
& u=2(\ln f)_{x x}, \\
& v=2(\ln f)_{x y}, \\
& w=2(\ln f)_{x t}, \\
& f=1+e^{\eta_{1}}+e^{\eta_{2}}+A_{12} e^{\eta_{1}+\eta_{2}}, \\
& A_{12} \gg 1
\end{aligned}
$$




\section{Type 2.}

$$
\begin{aligned}
& u=2(\ln f)_{x x}, \\
& v=2(\ln f)_{x y}, \\
& w=2(\ln f)_{x t}, \\
& f=1+e^{\eta_{1}}+e^{\eta_{2}}+A_{12} e^{\eta_{1}+\eta_{2}}, \\
& A_{12} \ll 1,
\end{aligned}
$$

where the other parameters are determined via Two-Soliton Solutions (6).

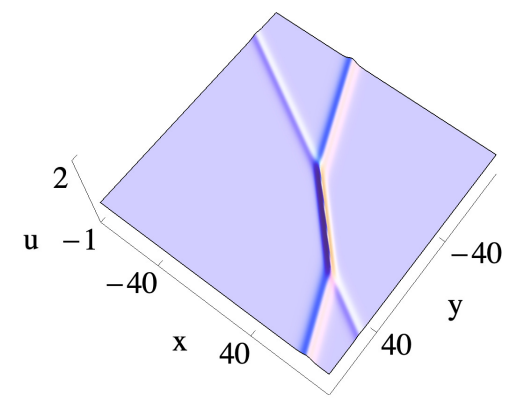

$\left(a_{1}\right) t=-3$

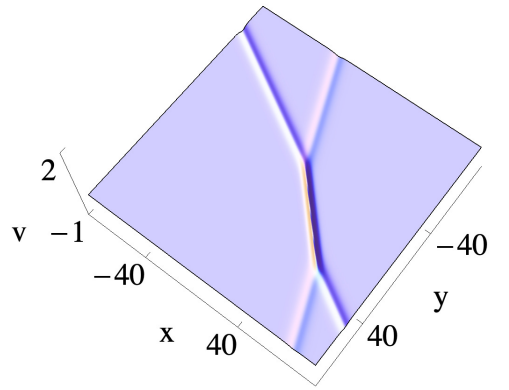

$\left(b_{1}\right) t=-3$

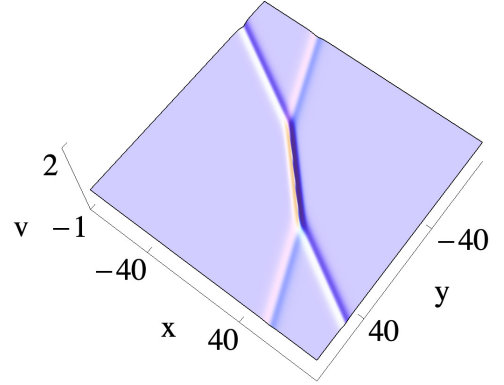

$\left(b_{2}\right) t=0$

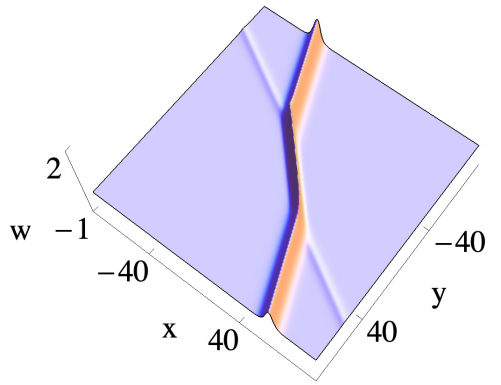

$\left(c_{2}\right) t=0$

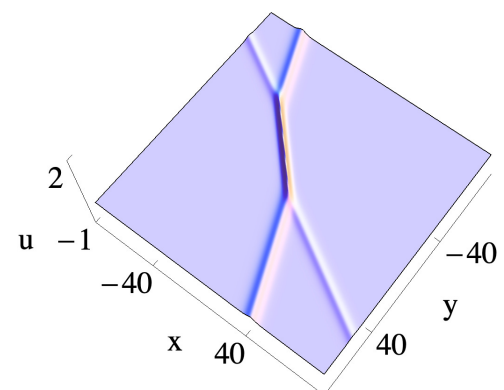

$\left(a_{3}\right) t=3$

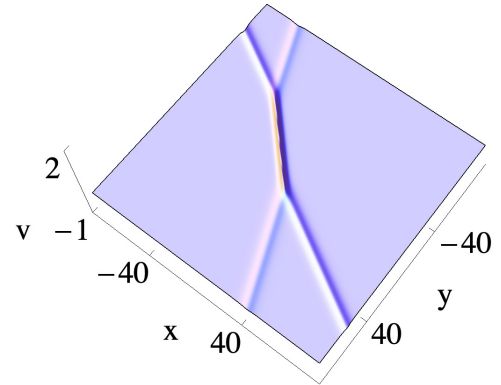

$\left(b_{3}\right) t=3$

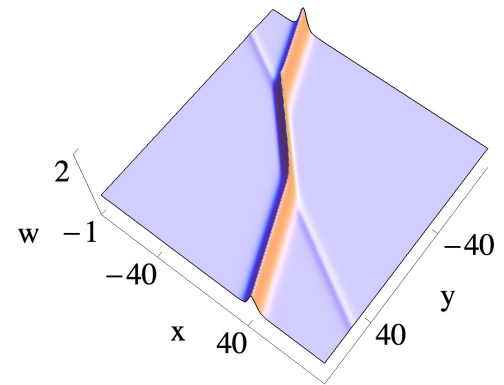

$\left(c_{3}\right) t=3$

Fig. 1 X-type soliton via Solutions (7) with $\alpha=1, k_{1}=-\frac{1}{2}, k_{2}=-\frac{1}{3}, p_{1}=\frac{1}{5}$, $p_{2}=\frac{35444429+\sqrt{1124999230000045}}{119999880}$ and $\zeta_{1}=\zeta_{2}=0$. 
For the shallow water waves, X-Type Soliton Solutions (7) and (8) are dependent on $\alpha$, the coefficient in System (2). Figures 1 show the X-type solitons via Solutions (7).

\section{Resonance Y-type soliton solutions for System (2)}

In order to construct some resonance soliton solutions which differ from those in Ref. [41], we take

$$
A_{\imath \jmath}=0, \quad(1 \leq \imath<\jmath \leq M, M<\imath<\jmath \leq N, N=M+L),
$$

in $N$-Soliton Solutions (5), namely,

$$
p_{\jmath}=\frac{-k_{\imath} k_{\jmath}\left[k_{\imath}\left(k_{\imath}-2 k_{\jmath}\right)\left(k_{\imath}-k_{\jmath}\right)-2 p_{\imath}\right] \pm \sqrt{-3 k_{\imath}^{3} k_{\jmath}^{2}\left(k_{\imath}-k_{\jmath}\right)^{2}\left(k_{\imath}^{3}+4 p_{\imath}\right)}}{2 k_{\imath}^{2}},
$$

which need to satisfy

$$
3 k_{\imath}^{3}\left(k_{\imath}^{3}+4 p_{\imath}\right)<0 .
$$

Taking $N$ as 2 or 3, our results are similar to those in Ref. [41]. However, taking $N=4$, we can derive a expression describing an interaction between two 2-resonance Y-type solitons for System (2), which are different from those in Ref. [41], i.e.,

$$
\begin{aligned}
& u=2(\ln f)_{x x}, \\
& v=2(\ln f)_{x y}, \\
& w=2(\ln f)_{x t}, \\
& f=1+e^{\eta_{1}}+e^{\eta_{2}}+e^{\eta_{3}}+e^{\eta_{4}}+A_{13} e^{\eta_{1}+\eta_{3}}+A_{14} e^{\eta_{1}+\eta_{4}}+A_{23} e^{\eta_{2}+\eta_{3}}+A_{24} e^{\eta_{2}+\eta_{4}},
\end{aligned}
$$

where

$$
\begin{aligned}
& p_{2}=\frac{-k_{1} k_{2}\left[k_{1}\left(k_{1}-2 k_{2}\right)\left(k_{1}-k_{2}\right)-2 p_{1}\right] \pm \sqrt{-3 k_{1}^{3} k_{2}^{2}\left(k_{1}-k_{2}\right)^{2}\left(k_{1}^{3}+4 p_{1}\right)}}{2 k_{1}^{2}} \\
& p_{4}=\frac{-k_{3} k_{4}\left[k_{3}\left(k_{3}-2 k_{4}\right)\left(k_{3}-k_{4}\right)-2 p_{3}\right] \pm \sqrt{-3 k_{3}^{3} k_{4}^{2}\left(k_{3}-k_{4}\right)^{2}\left(k_{3}^{3}+4 p_{3}\right)}}{2 k_{3}^{2}}
\end{aligned}
$$

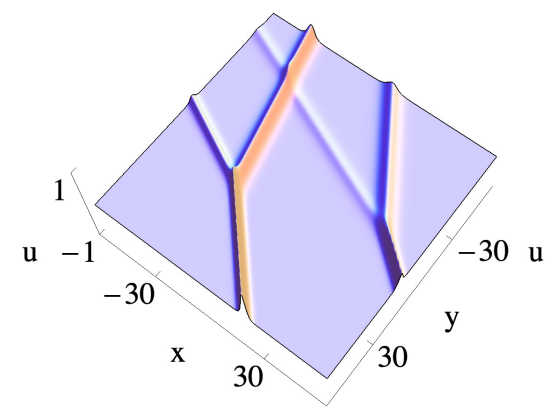

$$
\left(a_{1}\right) t=-10
$$

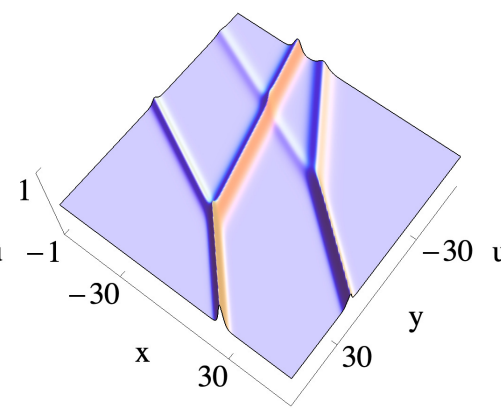

$\left(a_{2}\right) t=0$

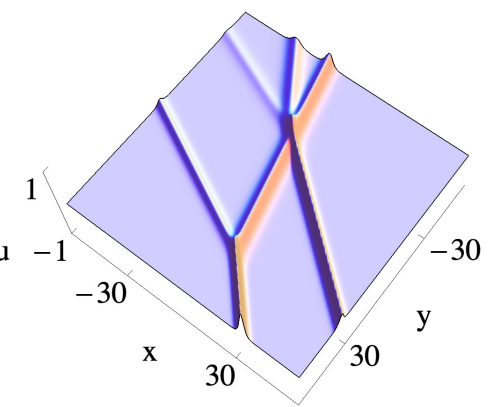

$\left(a_{3}\right) t=10$ 


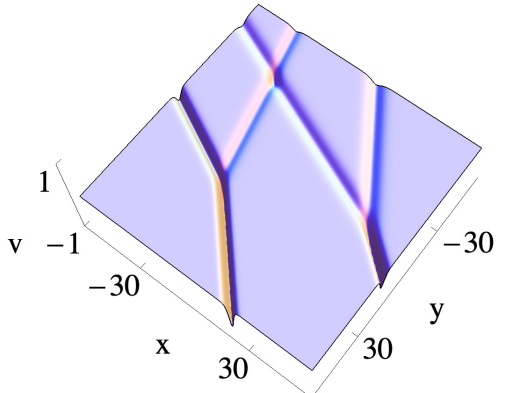

$\left(b_{1}\right) t=-10$

$\left(c_{1}\right) t=-10$

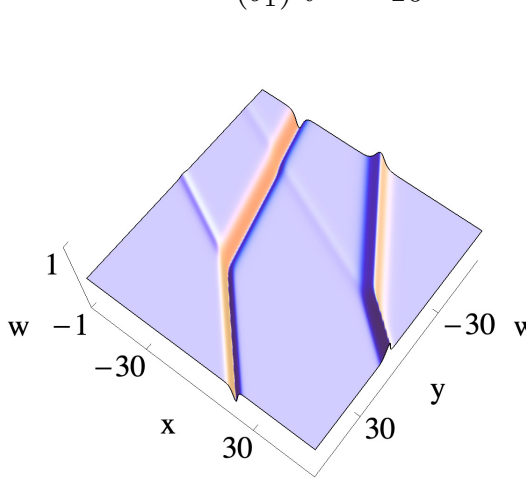

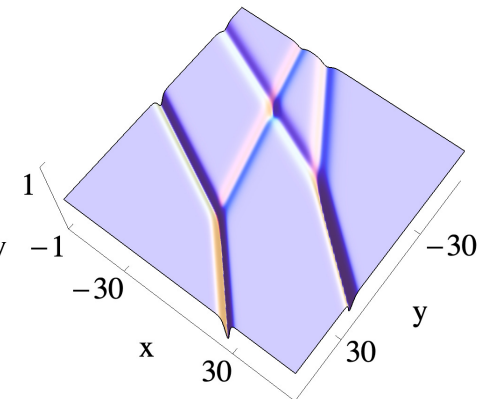

$\left(b_{2}\right) t=0$

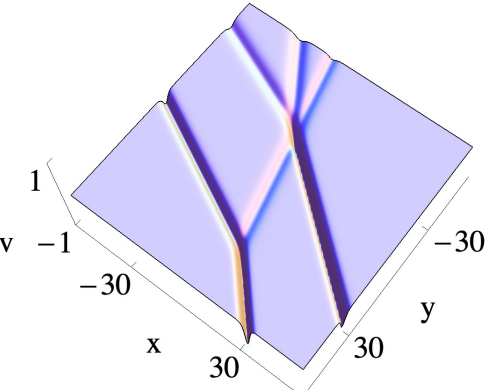

$\left(b_{3}\right) t=10$

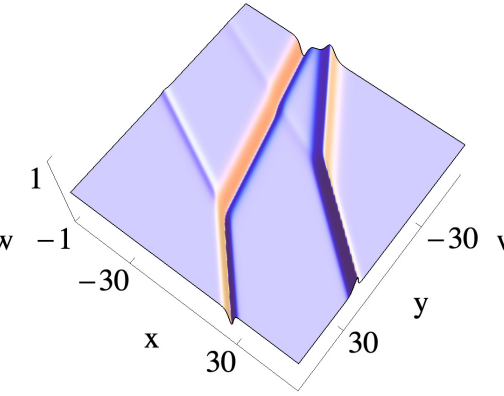

$\left(c_{2}\right) t=0$

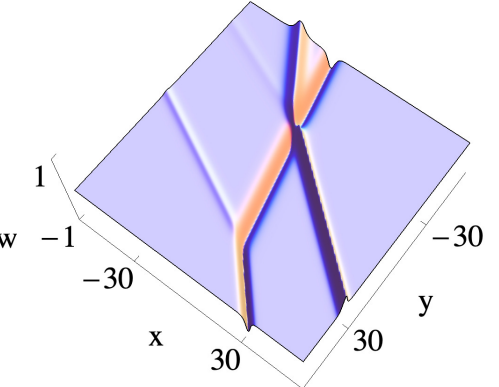

$\left(c_{3}\right) t=10$

Fig. 2 Interaction between the two 2-resonance Y-type solitons via Solutions (10) with $\alpha=\frac{1}{2}, k_{1}=-\frac{3}{10}$, $k_{2}=\frac{3}{5}, k_{3}=-\frac{1}{2}, k_{4}=\frac{4}{5}, p_{1}=\frac{7}{10}, p_{3}=1, \zeta_{1}=15, \zeta_{2}=-15, \zeta_{3}=-25$ and $\zeta_{4}=0$.

For the shallow water waves, Resonance Y-Type Soliton Solutions (10) rely on $\alpha$, the coefficient in System (2). Figure 2 displays the interaction between the two 2-resonance solitons based on Solutions (10).

\section{Hybrid solutions for System (2)}

4.1 Hybrid solutions consisting of the $M$-resonance Y-type solitons and $2 L$ solitons for System $(2)$

To find out the hybrid solutions consisting of the $M$-resonance Y-type solitons and $2 L$ solitons for System (2), we set the following conditions on $N$-Soliton Solutions (5):

$$
A_{\imath \jmath}=0, \quad \frac{k_{M+2 h-1}}{k_{M+2 h}}=\frac{p_{M+2 h-1}}{p_{M+2 h}} \neq \pm 1,(1 \leq \imath<\jmath \leq M, 1 \leq h \leq L, N=M+2 h) .
$$

Choosing $N=4$, i.e., $M=2, L=1, h=1$, we derive the hybrid solutions composed of the 
2-resonance Y-type solitons and two solitons for System (2) as

$$
\begin{aligned}
u= & 2(\ln f)_{x x}, \\
v= & 2(\ln f)_{x y}, \\
w= & 2(\ln f)_{x t}, \\
f= & 1+e^{\eta_{1}}+e^{\eta_{2}}+e^{\eta_{3}}+e^{\eta_{4}}+A_{13} e^{\eta_{1}+\eta_{3}}+A_{14} e^{\eta_{1}+\eta_{4}}+A_{23} e^{\eta_{2}+\eta_{3}}+A_{24} e^{\eta_{2}+\eta_{4}} \\
& +A_{34} e^{\eta_{3}+\eta_{4}}+A_{13} A_{14} A_{34} e^{\eta_{1}+\eta_{3}+\eta_{4}}+A_{23} A_{24} A_{34} e^{\eta_{2}+\eta_{3}+\eta_{4}},
\end{aligned}
$$

where

$$
\begin{aligned}
& k_{3}=\frac{k_{4} p_{3}}{p_{4}}, \\
& p_{2}=\frac{-k_{1} k_{2}\left[k_{1}\left(k_{1}-2 k_{2}\right)\left(k_{1}-k_{2}\right)-2 p_{1}\right] \pm \sqrt{-3 k_{1}^{3} k_{2}^{2}\left(k_{1}-k_{2}\right)^{2}\left(k_{1}^{3}+4 p_{1}\right)}}{2 k_{1}^{2}} .
\end{aligned}
$$

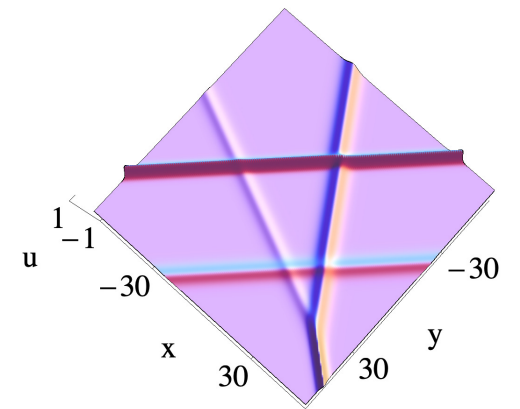

$\left(a_{1}\right) t=-10$

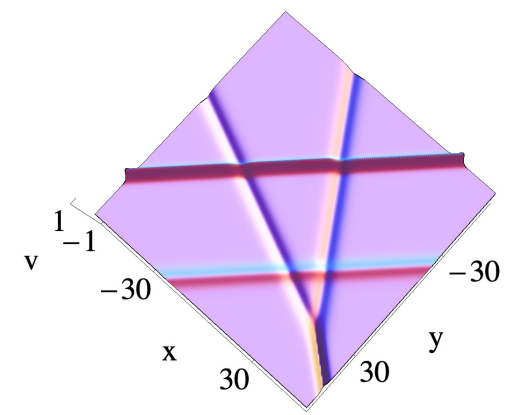

(b $\left.b_{1}\right) t=-10$

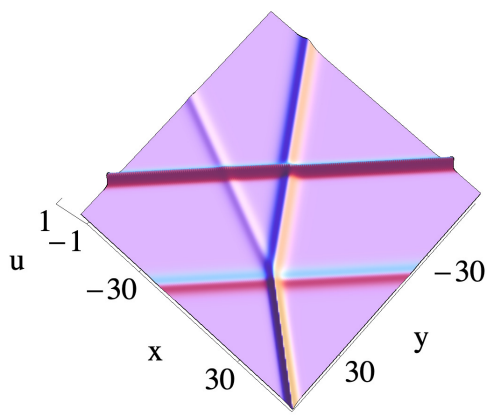

$\left(a_{2}\right) t=0$

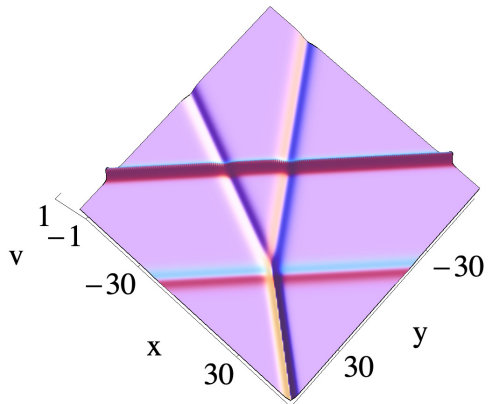

$\left(b_{2}\right) t=0$

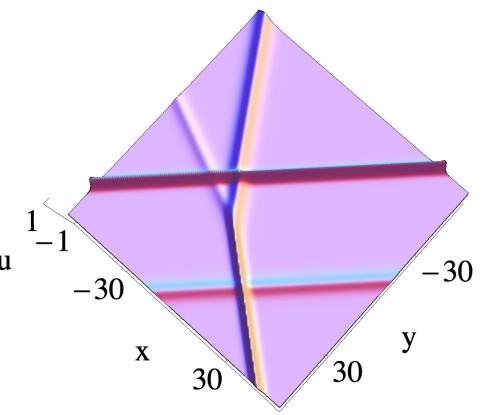

$\left(a_{3}\right) t=10$

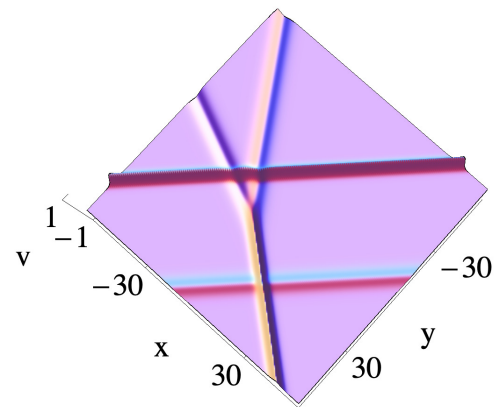

$\left(b_{3}\right) t=10$ 


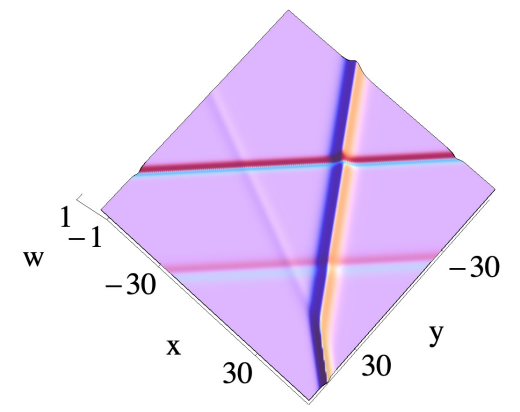

$\left(c_{1}\right) t=-10$

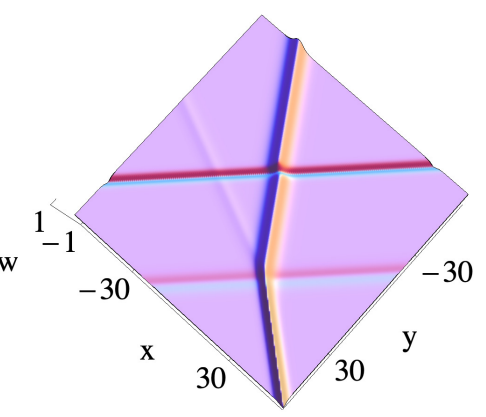

$\left(c_{2}\right) t=0$

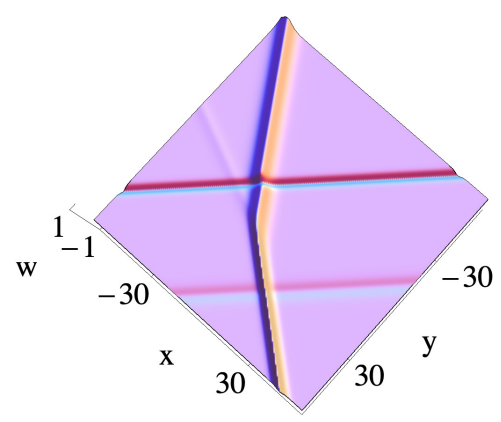

$\left(c_{3}\right) t=10$

Fig. 3 Interaction between the 2-resonance Y-type soliton and two solitons via Solutions (12) with $\alpha=\frac{1}{2}$, $k_{1}=-\frac{3}{10}, k_{2}=\frac{3}{5}, k_{4}=p_{4}=\frac{1}{2}, p_{1}=\frac{7}{10}, p_{3}=1, \zeta_{1}=-12, \zeta_{2}=0, \zeta_{3}=20$ and $\zeta_{4}=-20$.

For the shallow water waves, hybrid solutions composed of the 2-resonance Y-type solitons and two solitons, i.e., Solutions (12), depend on the coefficient $\alpha$ in System (2). Figure 3 displays the interaction between the 2-resonance Y-type soliton and two solitons based on Solutions (12).

4.2 Hybrid solutions consisting of the $M$-resonance Y-type solitons and higher-order breathers for System (2)

If $N$-Soliton Solutions (5) satisfy

$$
A_{\imath \jmath}=0, \quad \eta_{M+2 h-1}=\eta_{M+2 h}^{*}, \quad(1 \leq \imath<\jmath \leq M, 1 \leq h \leq R, N=M+2 h),
$$

we can derive the hybrid solutions consisting of the $M$-resonance Y-type solitons and $R$ thorder breathers for System (2), where the superscript “*” stands for the complex conjugation. Taking $N=4$, i.e., $M=2, R=1, h=1$, we obtain the hybrid solutions composed of the 2-resonance Y-type solitons and first-order breathers for System (2) as

$$
\begin{aligned}
u= & 2(\ln f)_{x x}, \\
v= & 2(\ln f)_{x y} \\
w= & 2(\ln f)_{x t}, \\
f= & 1+e^{\eta_{1}}+e^{\eta_{2}}+e^{\eta_{3}}+e^{\eta_{4}}+A_{13} e^{\eta_{1}+\eta_{3}}+A_{14} e^{\eta_{1}+\eta_{4}}+A_{23} e^{\eta_{2}+\eta_{3}}+A_{24} e^{\eta_{2}+\eta_{4}} \\
& +A_{34} e^{\eta_{3}+\eta_{4}}+A_{13} A_{14} A_{34} e^{\eta_{1}+\eta_{3}+\eta_{4}}+A_{23} A_{24} A_{34} e^{\eta_{2}+\eta_{3}+\eta_{4}},
\end{aligned}
$$

where

$$
\begin{aligned}
& k_{3}=k_{4}^{*}=k_{31}+i k_{32}, \quad p_{3}=p_{4}^{*}=p_{31}+i p_{32}, \quad \zeta_{3}=\zeta_{4}^{*}=\zeta_{31}+i \zeta_{32}, \\
& p_{2}=\frac{-k_{1} k_{2}\left[k_{1}\left(k_{1}-2 k_{2}\right)\left(k_{1}-k_{2}\right)-2 p_{1}\right] \pm \sqrt{-3 k_{1}^{3} k_{2}^{2}\left(k_{1}-k_{2}\right)^{2}\left(k_{1}^{3}+4 p_{1}\right)}}{2 k_{1}^{2}},
\end{aligned}
$$

$i=\sqrt{-1}$, while $k_{31}, k_{32}, p_{31}, p_{32}, \zeta_{31}$ and $\zeta_{32}$ are the real constants. 


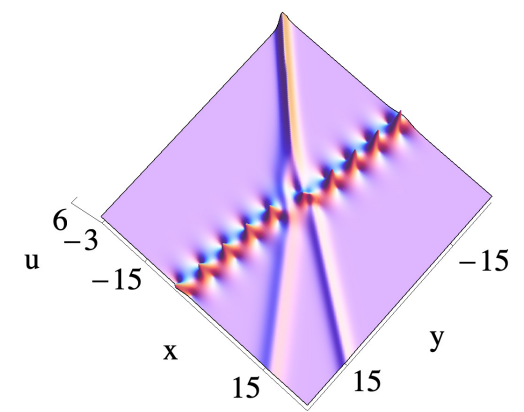

$\left(a_{1}\right) t=-1$

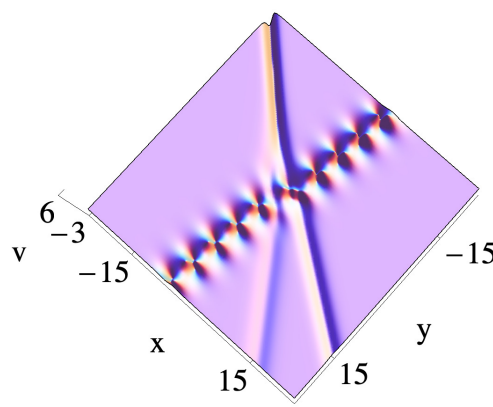

$\left(b_{1}\right) t=-1$

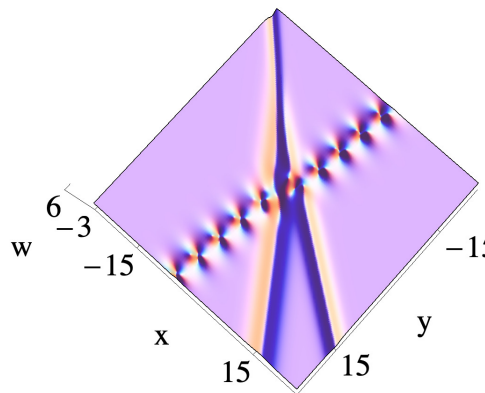

$\left(c_{1}\right) t=-1$

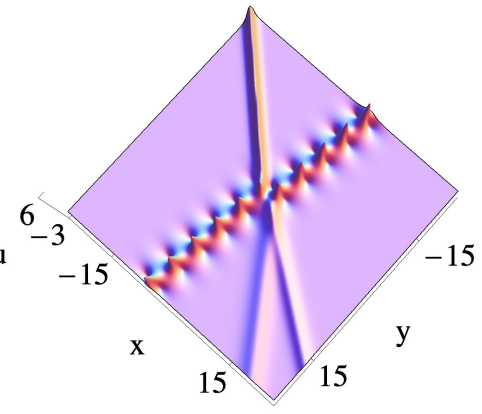

$\left(a_{2}\right) t=0$

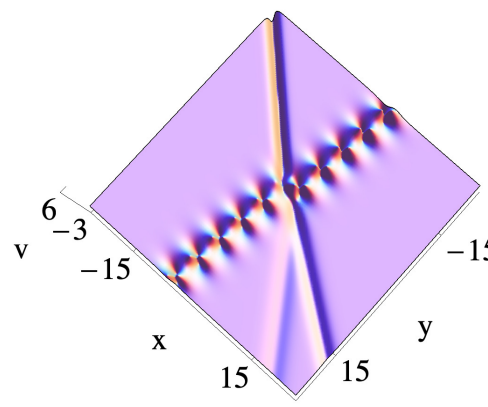

$\left(b_{2}\right) t=0$

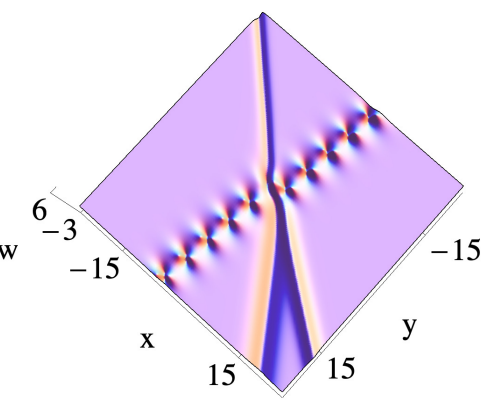

$\left(c_{2}\right) t=0$

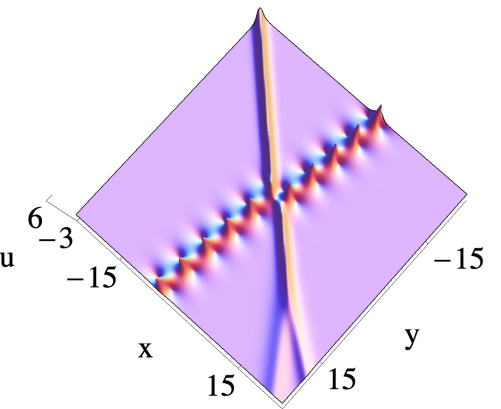

$\left(a_{3}\right) t=1$

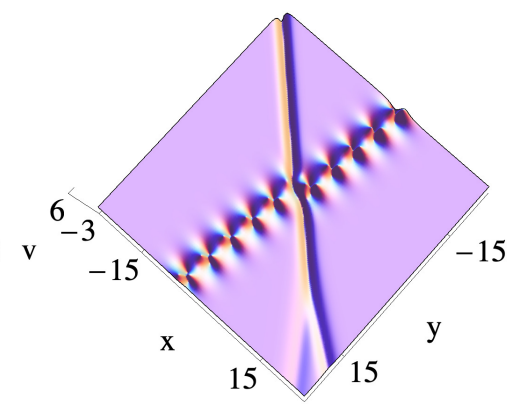

$\left(b_{3}\right) t=1$

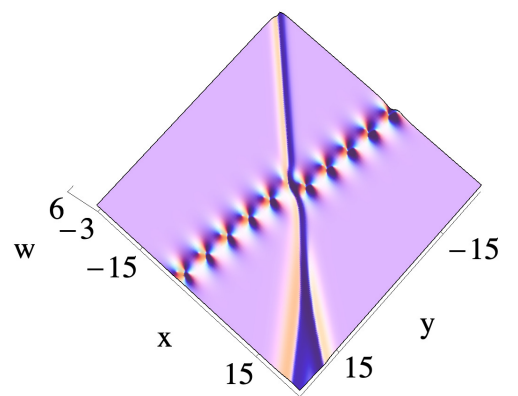

$\left(c_{3}\right) t=1$

Fig. 4 Interaction between the 2-resonance Y-type soliton and first-order breather via Solutions (10) with $\alpha=1, k_{1}=-1, k_{2}=1, k_{31}=\frac{4}{5}, k_{32}=-\frac{1}{5}, p_{1}=\frac{7}{10}, p_{31}=\frac{1}{10}, p_{32}=\frac{6}{5}$ and $\zeta_{1}=\zeta_{2}=\zeta_{31}=\zeta_{32}=0$.

For the shallow water waves, hybrid solutions composed of the 2-resonance Y-type solitons and first-order breathers, i.e., (14), rely on the coefficient $\alpha$ in System (2). Figure 4 exhibits the interaction between the 2-resonance Y-type soliton and first-order breather based on Solutions (14).

4.3 Hybrid solutions consisting of the $M$-resonance Y-type solitons and higher-order lumps for System (2) 
Setting the following conditions on $N$-Soliton Solutions (5):

$$
\begin{gathered}
k_{2 q-1}=k_{2 q}^{*}=K_{2 q-1} \varepsilon, \quad p_{2 q-1}=p_{2 q}^{*}=P_{2 q-1} \varepsilon, \quad \zeta_{2 q-1}=\zeta_{2 q}=\pi i, \quad \varepsilon \rightarrow 0, \quad A_{\imath \jmath}=0 \\
(2 Q<\imath<\jmath \leq N, 1 \leq q \leq Q, N=M+2 Q),
\end{gathered}
$$

we are able to obtain the hybrid solutions consisting of the $M$-resonance Y-type solitons and $Q$ th-order lumps for System (2). When we take $N=4$, i.e., $M=2, Q=q=1$, hybrid solutions composed of the 2-resonance Y-type solitons and first-order lumps for System (2) are derived as

$$
\begin{aligned}
u= & 2(\ln f)_{x x}, \\
v= & 2(\ln f)_{x y}, \\
w= & 2(\ln f)_{x t}, \\
f= & \theta_{1} \theta_{2}+B_{12}+\left(\theta_{1} \theta_{2}+B_{23} \theta_{1}+B_{13} \theta_{2}+B_{13} B_{23}+B_{12}\right) e^{\eta_{3}} \\
& \quad+\left(\theta_{1} \theta_{2}+B_{24} \theta_{1}+B_{14} \theta_{2}+B_{14} B_{24}+B_{12}\right) e^{\eta_{4}},
\end{aligned}
$$

where

$$
\begin{gathered}
B_{\imath \jmath}= \begin{cases}-\frac{6 K_{\imath}^{2} K_{\jmath}^{2}\left(K_{\imath} P_{\jmath}+K_{\jmath} P_{\imath}\right)}{\left(K_{\imath} P_{\jmath}-K_{\jmath} P_{\imath}\right)^{2}}, & 1 \leq \imath<\jmath<2 Q, \\
-\frac{6 K_{\imath}^{2} k_{\jmath}^{2}\left[K_{\imath}\left(k_{\jmath}^{3}+p_{\jmath}\right)+P_{\imath} k_{\jmath}\right]}{K_{\imath}^{2}\left(k_{\jmath}^{3}+p_{\jmath}\right)^{2}+K_{\imath} P_{\imath} k_{\jmath}\left(k_{\jmath}^{3}-2 p_{\jmath}\right)+P_{\imath}^{2} k_{\jmath}^{2}}, & 1 \leq \imath \leq 2 Q, \quad \jmath>2 Q,\end{cases} \\
\theta_{1}=K_{1} x+P_{1} y-\frac{\alpha K_{1}^{2}}{P_{1}^{2}} t, \quad \theta_{2}=K_{2} x+P_{2} y-\frac{\alpha K_{2}^{2}}{P_{2}^{2}} t, \\
K_{1}=K_{2}^{*}=K_{11}+i K_{12}, \quad P_{1}=P_{2}^{*}=P_{11}+i P_{12}, \quad \zeta_{1}=\zeta_{2}=\pi i, \\
p_{4}=\frac{-k_{3} k_{4}\left[k_{3}\left(k_{3}-2 k_{4}\right)\left(k_{3}-k_{4}\right)-2 p_{3}\right] \pm \sqrt{-3 k_{3}^{3} k_{4}^{2}\left(k_{3}-k_{4}\right)^{2}\left(k_{3}^{3}+4 p_{3}\right)}}{2 k_{3}^{2}},
\end{gathered}
$$

while $K_{11}, K_{12}, P_{11}$ and $P_{12}$ are the real constants.

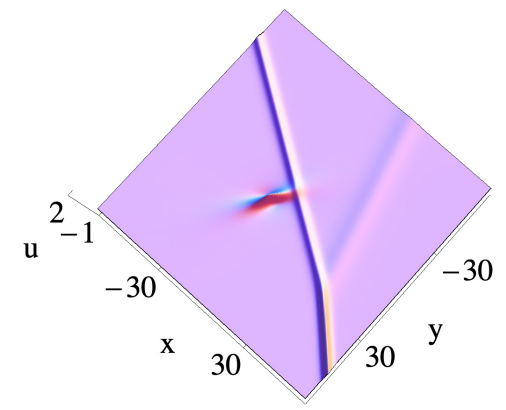

$\left(a_{1}\right) t=-10$

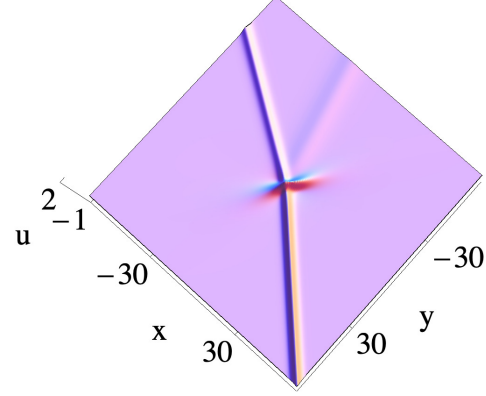

$\left(a_{2}\right) t=0$

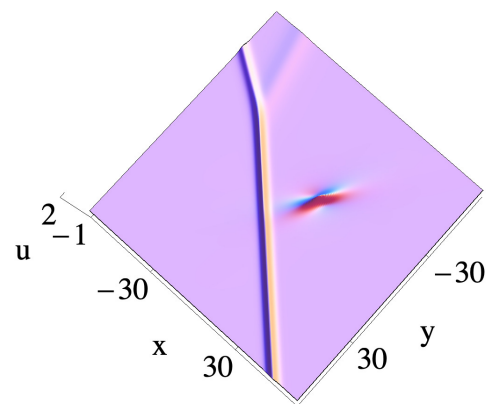

$\left(a_{3}\right) t=10$ 


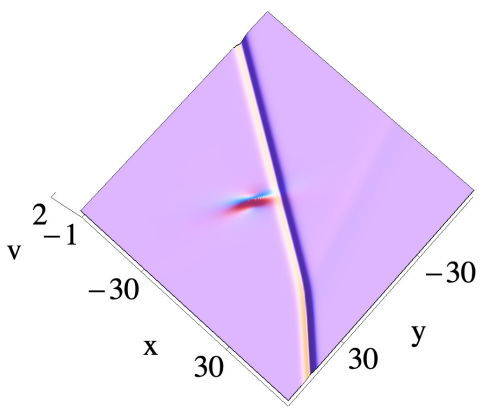

$\left(b_{1}\right) t=-10$

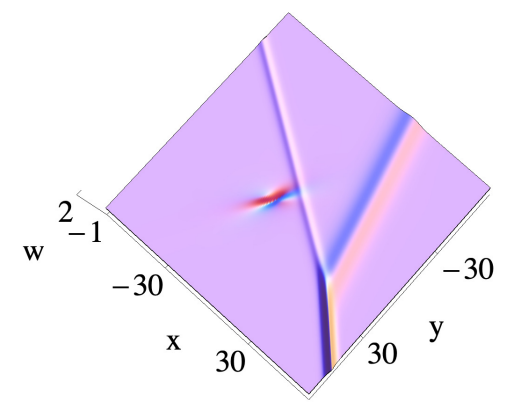

$\left(c_{1}\right) t=-10$

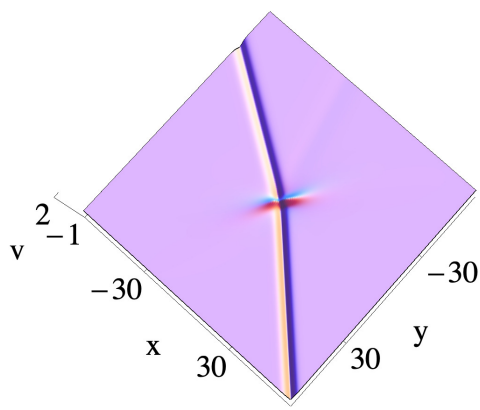

$\left(b_{2}\right) t=0$

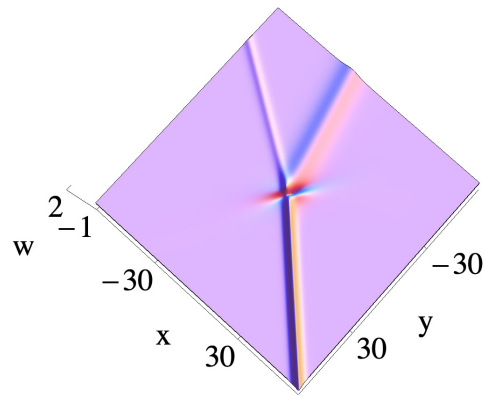

$\left(c_{2}\right) t=0$

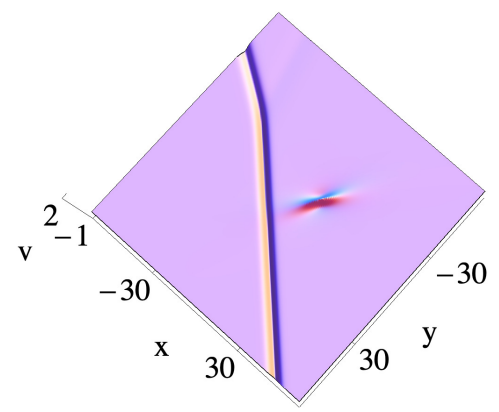

$\left(b_{3}\right) t=10$

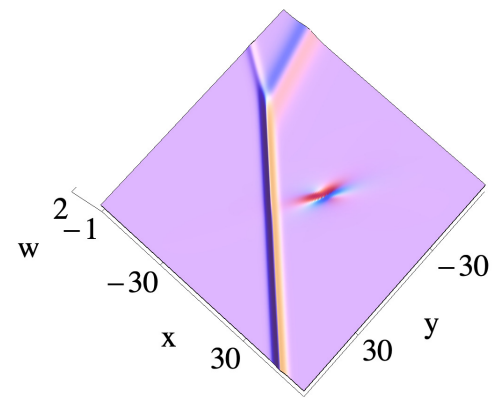

$\left(c_{3}\right) t=10$

Fig. 5 Interaction between the 2-resonance Y-type soliton and first-order lump via Solutions (16) with $\alpha=\frac{1}{2}, K_{11}=K_{12}=P_{11}=1, P_{12}=0, k_{3}=-\frac{1}{2}, k_{4}=\frac{1}{3}, p_{3}=\frac{3}{4}$ and $\zeta_{3}=\zeta_{4}=0$.

For the shallow water waves, hybrid solutions composed of the 2-resonance Y-type solitons and first-order lumps, i.e., (16), are dependent on the coefficient $\alpha$ in System (2). Figure 5 shows the interaction between the 2-resonance Y-type soliton and first-order lump via Solutions (16).

\section{Conclusions}

Since waves play a pervasive role in nature, people have paid attention to the study of waves, and to the water waves in particular. Shallow-water models have been applied in fluid dynamics, ocean dynamics, etc. For the shallow water waves, making use of the Hirota method and symbolic computation, we investigated a (2+1)-dimensional HSI system, i.e., System (2). Our results depend on $\alpha$, the coefficient in System (2). Main results obtained before can be summarized here:

- Two X-type soliton solutions, i.e., Solutions (7) and (8) have been obtained. Propagations of the X-type solitons have been displayed in Figs. 1. 
- Resonance Y-Type Soliton Solutions (10) describing the interaction between the two 2-resonance solitons have been derived. Figure 2 has shown the interaction between the two 2-resonance Y-type solitons.

- Hybrid solutions composed of the 2-resonance Y-type solitons and two solitons, the 2-resonance Y-type solitons and first-order breathers, and the 2-resonance Y-type solitons and first-order lumps, i.e., Solutions (12), (14) and (16), have been worked out. Interactions between the 2-resonance Y-type soliton and two solitons, the 2-resonance Y-type soliton and first-order breather, and the 2-resonance Y-type soliton and firstorder lump have been exhibited in Figs. 3, 4 and 5, respectively.

\section{Acknowledgments}

This work has been supported by the National Natural Science Foundation of China under Grant Nos. 11772017, 11272023 and 11471050, by the Fund of State Key Laboratory of Information Photonics and Optical Communications (Beijing University of Posts and Telecommunications), China (IPOC: 2017ZZ05) and by the Fundamental Research Funds for the Central Universities of China under Grant No. 2011BUPTYB02.

\section{Declaration of Competing Interest}

The authors declare that they have no known competing financial interests or personal relationships that could have appeared to influence the work reported in this paper.

\section{Data Availability Statement}

This paper has no associated data or the data will not be deposited.

\section{References}

[1] Ankiewicz A.: Rogue and semi-rogue waves defined by volume, Nonlinear Dyn. 104, 4241-4252 (2021)

[2] Dostal L., Hollm M., Kreuzer E.: Study on the behavior of weakly nonlinear water waves in the presence of random wind forcing, Nonlinear Dyn. 99, 2319-2338 (2020)

[3] Hascoët R., Raillard N., Jacques N.: Effect of forward speed on the level-crossing distribution of kinematic variables in multidirectional ocean waves, Ocean Eng. 235, 109345 (2021) 
[4] Ibrahim R.A.: Assessment of breaking waves and liquid sloshing impact, Nonlinear Dyn. 100, 1837-1925 (2021)

[5] Hogan P. A., Puetzfeld D.: Gravitational waves with colliding or noncolliding wave fronts, Phys. Rev. D 103, 124064 (2021)

[6] Steer J.N., Borthwick A.G.L., Onorato M., Chabchoub A., Bremer T. S.: Hydrodynamic $X$ waves, Phys. Rev. Lett. 123, 184501 (2019)

[7] Duran A., Richard G.L.: Modelling coastal wave trains and wave breaking, Ocean Model. 147, $101581(2020)$

[8] Blank D., Morgan J., Caniven Y.: Geometrically controlled slow slip enhanced by seismic waves: A mechanism for delayed triggering, Earth Planet. Sci. Lett. 554, 116695 (2021)

[9] Huller S., Raj G., Rozmus W., Pesme D.: Crossed beam energy transfer in the presence of laser speckle ponderomotive self-focusing and nonlinear sound waves, Phys. Plasmas 27, $022703(2020)$

[10] Shim H., Chung H., Miller O.D.: Maximal free-space concentration of electromagnetic waves, Phys. Rev. Appl. 14, 014007 (2020)

[11] Bliokh K.Y., Leykam D., Lein M., Nori F.: Topological non-Hermitian origin of surface Maxwell waves, Nat. Commun. 10, 580 (2019)

[12] Nettel S.: Wave Physics, Springer, Berlin, (2003)

[13] Debnath L.: Nonlinear Water Waves, Academic Press, Boston, (1994)

[14] Slunyaev A.V.: Group-wave resonances in nonlinear dispersive media: The case of gravity water waves, Phys. Rev. E 97, 010202 (2018)

[15] Khusnutdinova K.R., Stepanyants Y.A., Tranter M.R.: Soliton solutions to the fifthorder Korteweg-de Vries equation and their applications to surface and internal water waves, Phys. Fluids 30, 022104 (2018)

[16] Wazwaz A.M.: Two new Painlevé integrable KdV-Calogero-Bogoyavlenskii-Schiff (KdVCBS) equation and new negative-order KdV-CBS equation, Nonlinear Dyn. 104, 4311$4315(2021)$

[17] Peng L.J.: Different wave structures for the completely generalized Hirota-Satsuma-Ito equation, Nonlinear Dyn. (2021). https://doi.org/10.1007/s11071-021-06602-0 
[18] Marchukov O.V., Malomed B.A., Dunjko V., Ruhl J., Olshanii M., Hulet R.G., Yurovsky V.A.: Quantum fluctuations of the center of mass and relative parameters of nonlinear Schrödinger breathers, Phys. Rev. Lett. 125, 050405 (2020)

[19] Sulaiman T.A., Yusuf A., Alquran M.: Dynamics of optical solitons and nonautonomous complex wave solutions to the nonlinear Schrödinger equation with variable coefficients, Nonlinear Dyn. 104, 639-648 (2021)

[20] Selima E.S., Yao X., Wazwaz A.M.: Multiple and exact soliton solutions of the perturbed Korteweg-de Vries equation of long surface waves in a convective fluid via Painlevé analysis, factorization, and simplest equation methods, Phys. Rev. E 95, 062211 (2017)

[21] Cheemaa N., Seadawy A.R., Sugati T.G., Baleanu D.: Study of the dynamical nonlinear modified Korteweg-de Vries equation arising in plasma physics and its analytical wave solutions, Results Phys. 19, 103480 (2020)

[22] Tang X.Y., Cui C.J., Liang Z.F., Ding W.: Novel soliton molecules and wave interactions for a $(3+1)$-dimensional nonlinear evolution equation, Nonlinear Dyn. (2021). https://doi.org/10.1007/s11071-021-06687-7

[23] Liu J.G., Zhu W.H.: Breather wave solutions for the generalized shallow water wave equation with variable coefficients in the atmosphere, rivers, lakes and oceans, Comput. Math. Appl. 78, 848 (2019)

[24] Kumar V., Wazwaz A.M.: Lie symmetry analysis for complex soliton solutions of coupled complex short pulse equation, Math. Meth. Appl. Sci. 44, 5238 (2021)

[25] Gao X.Y., Guo Y.J., Shan W.R.: Optical waves/modes in a multicomponent inhomogeneous optical fiber via a three-coupled variable-coefficient nonlinear Schrödinger system, Appl. Math. Lett. 120, 107161 (2021)

[26] Ma W.X., Batwa S.: A binary Darboux transformation for multicomponent NLS equations and their reductions, Anal. Math. Phys. 11, 44 (2021)

[27] Prado H.P., Cisneros-Ake L.A.: The direct method for multisolitons and two-hump solitons in the Hirota-Satsuma system, Phys. Lett. A 384, 126471 (2020)

[28] Li J., Chen Q., Li B.: Resonance Y-type soliton solutions and some new types of hybrid solutions in the $(2+1)$-dimensional Sawada-Kotera equation, Commun. Theor. Phys. 73, 045006 (2021) 
[29] Ma H.C., Cheng Q.X., Deng A.P.: Soliton molecules and some novel hybrid solutions for the (2+1)-dimensional generalized Konopelchenko-Dubrovsky-Kaup-Kupershmidt equation, Commun. Theor. Phys. 72, 095001 (2020)

[30] Jin Y.T., Chen A.H.: Resonant solitary wave and resonant periodic wave solutions of the Kudryashov-Sinelshchikov equation, Phys. Scr. 95, 085208 (2020)

[31] Kayum M.A., Akbar M.A., Osman M.S.: Stable soliton solutions to the shallow water waves and ion-acoustic waves in a plasma, Wave Random Complex. (2020). https://doi.org/10.1080/17455030.2020.1831711

[32] Kurtz F., Ropers C., Herink G.: Resonant excitation and all-optical switching of femtosecond soliton molecules, Nat. Photonics 14, 9 (2019)

[33] Lashkin V.M.: Perturbation theory for solitons of the Fokas-Lenells equation: Inverse scattering transform approach, Phys. Rev. E 103, 042203 (2021)

[34] Kako F., Yajima N.: Interaction of ion-acoustic solitons in two-dimensional space, J. Phys. Soc. Jpn. 49, 2063 (1980)

[35] Lou S.Y.: Soliton molecules and asymmetric solitons in three fifth order systems via velocity resonance, J. Phys. Commun. 4, 041002 (2020)

[36] Hirota R.: The Direct Method in Soliton Theory, Cambridge Univ. Press, New York, (2004)

[37] Zhou Y., Manukure S.: Complexiton solutions to the Hirota-Satsuma-Ito equation, Math. Meth. Appl. Sci., 42, 12344 (2019)

[38] Liu Y., Wen X.Y., Wang D.S.: The N-soliton solution and localized wave interaction solutions of the (2+1)-dimensional generalized Hirota-Satsuma-Ito equation, Comput. Math. Appl. 77, 947 (2019)

[39] Zhou Y., Wang C., Zhang X.: Rational localized waves and their Absorb-Emit interactions in the (2+1)-dimensional Hirota-Satsuma-Ito equation, Mathematics 8, 1807 (2020)

[40] Liu J.G., Zhu W.H., Zhou L.: Multi-wave, breather wave, and interaction solutions of the Hirota-Satsuma-Ito equation, Eur. Phys. J. Plus 135, 20 (2020)

[41] Kuo C.K., Ma W.X.: A study on resonant multi-soliton solutions to the (2+1)dimensional Hirota-Satsuma-Ito equations via the linear superposition principle, Nonlinear Anal. 190, 111592 (2020) 
[42] Liu W., Wazwaz A.M., Zheng X.X: High-order breathers, lumps, and semi-rational solutions to the (2+1)-dimensional Hirota-Satsuma-Ito equation, Phys. Scr., 94, 075203 (2019)

[43] Zhou Y., Manukure S., Ma W.X.: Lump and lump-soliton solutions to the HirotaSatsuma-Ito equation, Commun. Nonlinear Sci. Numer. Simulat. 68, 56 (2019)

[44] Shen W., Ma Z., Fei J., Zhou Q.: Novel characteristics of lump and lump-soliton interaction solutions to the $(2+1)$-dimensional Alice-Bob Hirota-Satsuma-Ito equation, Mod. Phys. Lett. B 34, 2050419 (2020) 